\title{
The global effect: what determines where the eyes land?
}

\begin{abstract}
Stefan Van der Stigchel
Helmholtz Institute, Utrecht University

\author{
Tanja C.W. Nijboer \\ Helmholtz Institute, Utrecht University
}

In certain situations, the endpoint of an eye movement is not positioned on the centre of a target element, but deviates in the direction of another element. This phenomenon has been termed 'the global effect' and has proven to constitute a valuable measure of various processes that control and influence our oculomotor behavior. The goal of the current review is to provide insight in the factors that determine where the eyes land. We will focus on the fundamental characteristics of the global effect and discuss the various domains in which the global effect has been applied. The global effect appears to be best explained in terms of a weighted average of activity in a saccade map.
\end{abstract}

\section{Keywords: eye movements, global effect, saccade averaging, reading, visual search, review}

\section{Introduction}

In order to explore our environment, we continuously make fast eye movements called saccades. Saccades are the movements of the eyes made to point the fovea onto an object for detailed visual analysis. In order to successfully complete a task in our dynamic visual environment, it is crucial to make saccades to locations that are relevant for the task demands. For instance, while driving a car, it is essential to keep your eyes on the road and to fixate landmarks that might guide steering. However, this process also constitutes ignoring the parts of the visual scene that are distracting for completing the task. So, while driving, it is important not to fixate elements that might interfere with driving, like flashing billboards located along the route. When a saccade is executed, its endpoint therefore reflects the outcome of a selection process of all possible target locations.

Various studies that have investigated the exact landing position of eye movements have observed that in certain situations, the endpoint of an eye movement is not positioned on the centre of an element, but deviates in the direction of another element. This phenomenon has been termed 'the global effect'. The aim of the current review is to discuss these studies and to provide insight in the factors that determine where the eyes land. There are earlier reviews on aspects of the global effect (Findlay \& Walker, 1999, Vitu, 2008), but the aim of the present review is to present a complete and up-to-date overview of the literature. First, we will focus on the fundamental characteristics of the global effect, after which we will discuss the various domains in which the global effect has been observed.

The fundamental idea with respect to the global effect is that eye movements tend to land on the centre of gravity in the visual field (Coren \& Hoenig, 1972). This centre of gravity then reflects the relative saliency of the different elements in the visual scene. If more than one element is presented, this relative saliency is not positioned on one element, but might be positioned on an intermediate location. In this situation, the saccade will be directed to this intermediate location.

\section{History of the global effect}

The first suggestion in the literature with respect to the global effect was made in a theoretical paper from 1947 (Pitts \& McCulloch, 1947). Pitts and McCulloch 
described possible neural mechanisms that could recognize auditory and visual forms. When discussing the visual system, they propose that the oculomotor system computes "the centre of gravity of the distribution of brightness". The system then "supplies impulses at a rate proportional to these coordinates to the lateral and vertical eye-muscles in such a way that these then turn the visual axis towards the centre of gravity" (p. 137).

Although it had been reported earlier that participants have a tendency towards fixating in the "centre of gravity" of a stimulus array (Kaufman \& Richards, 1969), the first systematic experimental evidence for this suggestion was provided by Coren and Hoenig (1972). Besides the observation by Lévy-Schoen (1969) that saccade latency is increased in the presence of an additional element, it was unknown whether non-targets influence the saccade to the target. To investigate whether non-targets influence the length of a saccade to a target, a red target was presented to the left or the right of the fixation point. Simultaneously, additional elements could be presented at the same axis as the target. Results showed that the length of the saccade was influenced by the additional stimuli. The saccade was longer when nontargets were presented beyond the target, whereas the saccade was shorter when non-targets were presented in between the target and the fixation point. Two follow-up experiments were performed. When only one non-target was presented, it was shown that the effect is strongest when the non-target is presented in between the target and the fixation point and the distance between the two elements was small. Furthermore, the effect was still present, although less pronounced, when the target was more difficult to identify and more precise saccades were required.

After the publication of this study, it took 10 years before this phenomenon was examined further (Findlay, 1982). John Findlay introduced the term 'global effect' in a study which can be considered the start of a period of extensive research on the global effect. The various conclusions of this study will be discussed throughout this review.

\section{The influence of stimulus properties on the global effect}

When interpreting the literature on the global effect, there are four stimulus properties that are known to influence the landing position of an eye movement when multiple elements are presented:

Stimulus location: As mentioned, Coren and Hoenig (1972) already revealed that the deviation of the saccade endpoint in the direction of the non-target is strongest when the non-target is positioned in between the target and central fixation. This finding has later been replicated (e.g. Findlay, 1982) and might be related to the cortical magnification factor (Coeffe \& O'Regan, 1987, Findlay, 1982, Vitu, 1991). Because of this factor, elements presented closer to fixation might be more potent for the oculomotor system (Van der Stigchel, Meeter \& Theeuwes, 2007, Vitu, Lancelin, Jean \& Farioli, 2006), resulting in a stronger attraction of the saccade endpoint.

Stimulus size: When two stimuli are presented, the saccade endpoint deviates in the direction of the largest stimulus (Findlay, 1982). In the study by Findlay, elements were presented on the horizontal axis and there was no specific target element. It has to be noted that this experiment only tested a limited range of stimulus sizes: large being a square of $0.42^{\circ}$ and small being a square of $0.14^{\circ}$ (see also Findlay, Brogan \& Wenban-Smith, 1993).

Stimulus intensity: The saccade endpoint is positioned closer to the stimulus with the strongest (brightest) intensity (Deubel, Wolf \& Hauske, 1984). Results of an experiment in which two visual elements were presented with different intensities on the horizontal axis showed a linear relationship between the saccade endpoint and the relative target intensities.

Similarity to the background: The influence of the stimulus properties described above suggests that the saccade endpoint is positioned towards the element which is the most potent in the visual field. This is consistent with findings from an experiment which used a complex visual scene, in which elements differed from the background only by the orientation of its constituent elements (Deubel, Findlay, Jacobs \& Brogan, 1988). In this experiment, the saccade endpoint was positioned closer to the element which had the strongest dissimilarity to the background.

\section{The influence of stimulus distance on the global effect}

All studies on the global effect discussed until now have presented the stimuli on the horizontal axis. In these studies, the global effect was only observed when the 
stimuli were presented in the same left or right visual field. When both elements were presented in the same visual field, but with different directions, it was observed that the distance between the two elements is a crucial factor in determining whether a global effect was observed (Ottes, van Gisbergen \& Eggermont, 1984). The advantage of this approach is that both elements have the same distance to the fixation point, eliminating a contributing influence of this factor. Results showed that the global effect was mainly observed when the distance between the stimuli was small $\left(30^{\circ}\right.$ angular distance), whereas a bimodal response pattern was observed when the distance was large $\left(90^{\circ}\right.$ angular distance). In this situation, a bimodal response pattern indicates that little averaging occurred, but that saccades were predominantly executed in the direction of one of the two stimuli (see also Ottes, Van Gisbergen \& Eggermont, 1985).

In a more systematic investigation of the influence of the distance between the two elements, it was revealed that there was a specific zone in which the global effect occurred (Walker, Deubel, Schneider \& Findlay, 1997). In a series of experiments, Walker and colleagues examined the influence of a distractor on an eye movement to a target on the horizontal axis. Only when the distractor was presented in a zone of around $20^{\circ}$ angular distance, the amplitude of the saccade was influenced. Interestingly, saccade latency was unaffected in this condition. Outside this zone, amplitude was not influenced by the distractor, but saccade latencies increased when compared to a condition in which no distractor was presented ('the remote distractor effect').

On the basis of these results, it can be concluded that the global effect predominantly occurs when the two elements are presented in close proximity. This finding has later been confirmed (Chou, Sommer \& Schiller, 1999, Van der Stigchel \& Theeuwes, 2005), although various studies have also shown that some deviation of saccade endpoint in the direction of a non-target still occurs when stimulus distance is larger (Arai, McPeek \& Keller, 2004, Van der Stigchel, de Vries, Bethlehem \& Theeuwes, 2011, Van der Stigchel, Mulckhuyse \& Theeuwes, 2009). These latter studies have not investigated whether the endpoint distribution was unimodal or bimodal, however. It could be that 'true averaging', in which a unimodal distribution of endpoints is observed, is restricted to certain distance between the two elements. In other situations, a shift of saccade endpoint in the direction of the additional stimulus can occur, but the endpoint distribution will be bimodal.

\section{The influence of saccade latency on the global effect}

Although it was already observed in an earlier study that the global effect is more pronounced for saccades with a short latency (Findlay, 1982), Ottes and colleagues (1985) were the first to systematically investigate the influence of saccade latency on the global effect. In their Experiment 2, in which a saccade had to be made to a target in the presence of a non-target, participants were given the instruction to emphasize either speed or accuracy. Results showed that the only way to avoid the global effect was to wait $300 \mathrm{~ms}$ to initiate a saccade. When the latency was shorter, the task instruction to fixate the target hardly influenced the saccade endpoint and the saccade was predominantly executed to an intermediate location between the target and the nontarget.

This influence of saccade latency was confirmed in a later study in which the moment of saccade initiation was delayed (Coeffe \& O'Regan, 1987). Participants were required to remain fixated on the central fixation mark until it disappeared. When the fixation mark disappeared after the visual stimuli were presented on the screen, the global effect was smaller compared to a situation in which the visual stimuli were presented simultaneously with the disappearance of the fixation mark.

The important contribution of saccade latency was also observed in a visual search task in which the number of items in the display was varied (McSorley \& Findlay, 2003). When only two items were presented (a target and a non-target) a clear global effect was observed. However, when more items were presented, accuracy of the saccade to the target increased, which could be attributed to an increased saccade latency due to the presence of multiple items in the display.

In monkeys, it has been shown that the global effect is associated with express saccades, which have an extremely short latency (< $100 \mathrm{~ms}$ ) (Chou et al., 1999). Express saccades are thought to occur within the fastest time possible for a visual stimulus to be translated into the target of a saccade (Dorris, Pare \& Munoz, 1997, Pare 
\& Munoz, 1996) and they are proposed to constitute purely reflexive movements (Fischer \& Weber, 1993). For the tested monkeys, there was a higher probability of express saccades being averaged than regular saccades. This global effect for express saccades occurred even when both elements were separated $45^{\circ}$ of angular distance (Edelman \& Keller, 1998).

Given the modulating role of saccade latency on the global effect, saccade latency is an important factor when interpreting the results of an experimental study. For instance, when a certain manipulation increases saccade latency and also abolishes the global effect, the absence of the global effect might simply be due to the increased saccade latency. The best comparison with respect to the global effect is therefore between conditions that have a similar saccade latency.

\section{The influence of higher-order signals on the global effect}

The papers discussed until now have unraveled the effects of lower-order properties on the global effect. This might suggest that the global effect is automatic and is not influenced by higher-order signals, like task instructions. Indeed, early studies claimed that task instructions did not influence the global effect (Menz \& Groner, 1987, Ottes et al., 1985), because the global effect was still observed when one of the two elements was designated as the target element and the other element as a non-target element.

A first hint that higher-order signals can influence the global effect was provided by a study in which the target location was kept constant during a block of trials (Coeffe \& O'Regan, 1987). Stimuli consisted of a string of letters within which a target letter was indicated by two plus signs (one above and one below it) that were presented on the horizontal axis. By keeping the target location constant, the observer could predict the likely location of the target. Results showed that this expectancy decreased the size of the global effect; eye movements were more accurately initiated towards the target location, although the global effect was not completely abolished when the location was kept constant.

The finding that the global effect is not automatic, but can be influenced by higher-order signals was extended by He and Kowler (1989). By varying the probability of the target appearing in one of two locations, they showed that saccades were biased towards the most likely target location, regardless of where the target was actually presented. This indicates that the endpoint of an eye movement is based on prior history of target locations and expectancies about the future location of the target. Moreover, the effect of probability was reduced when the discrimination of the target from the non-target was easier. With easier discrimination, subject did not need to rely on information about the likely location of the target.

The global effect also becomes less pronounced when information is given on a trial to trial basis about where the target is going to be presented (Aitsebaomo \& Bedell, 2000). This study decreased the ambiguity about which element was the target by presenting a prior auditory cue in advance of each trial that provided unambiguous information about which target represent the saccadic goal. A high pitch indicated that the observer should make an eye movement to the more distant of the doubletarget pair, whereas a low pitch indicated that the nearer element was the target location.

Additional evidence of the influence of higher-order signals on the global effect comes from studies which have compared conditions with and without specific task instructions. For instance, the global effect was shown to be more prominent when no specific instruction was given regarding the saccade target compared to a condition in which a specific target element was specified (Chou et al., 1999). Also, when the generation of accurate saccades is a task requirement, the global effect can be completely abolished (Findlay \& Blythe, 2009) or the zone in which the global effect is observed can be modulated (Findlay \& Kapoula, 1992). It has to be noted that some of these manipulations also increased saccade latencies, making it difficult to isolate the contribution of the experimental manipulation on the global effect itself, as explained in the previous section.

\section{The global effect in other modalities}

All discussed studies have used visual stimuli to investigate the saccade endpoint in the presence of multiple stimuli. There is, however, evidence that auditory stimuli can also deviate the endpoint of an eye movement. Frens and colleagues showed that when visual and auditory stimuli were presented temporally aligned, 
saccades typically started in a direction in between the two stimuli (Frens, Van Opstal \& Van der Willigen, 1995). This only happened when their spatial separation was not too large. The presence of a localized auditory stimulus can thus influence the endpoint of a saccade to a visual target when they are placed close enough, in a similar way as a visual stimulus.

Interestingly, the global effect is not restricted to eye movements, but can also be observed for other motor movements, like reaching movements (Sailer, Eggert, Ditterich \& Straube, 2002). In this study, eye and hand movements were measured simultaneously while participants were required to move the eye and the hand to a peripheral target in the presence of a non-target. In line with evidence that the eye and the hand are tightly coupled (e.g. Gielen, van den Heuvel \& van Gisbergen, 1984), results showed that the endpoint of the hand movement deviated in the same direction as the eye movement, namely in the direction of the non-target. In various conditions, however, the magnitude of the global effect was different for eye and hand movements, which led the authors to claim that the coupling between the eye and the hand during target selection is not achieved through a shared target representation.

The global effect is not only observed when two elements are presented at the time of the saccade initiation. It is also observed when one of the elements has to be kept in memory (Herwig, Beisert \& Schneider, 2010). In the task by Herwig and colleagues, participants had to memorize a target location for a subsequent memory-guided saccade. During the retention interval, an irrelevant element was briefly flashed on a portion of the trials. These irrelevant elements attracted the saccade's landing position in that a global effect was observed when it was presented close to the location of a memory location. This result showed that keeping a location in spatial working memory is achieved through the continuous activation of the corresponding location in the oculomotor system.

\section{The global effect in special populations}

With the high number of studies using the global effect as a measure, it is surprising that there is very little work on the global effect in special populations. There is one study from 1978 which used the classic target/nontarget paradigm on the horizontal axis to show that the global effect is also observed in a group of children with a mean age of 8.5 years (Cohen \& Ross, 1978). There is no systematic study besides this one experiment, which only used a limited number of participants (nine in both the adult and the young group) and only tested thirdgraders.

In a completely different domain, endpoint deviations induced by a non-target were investigated in patients with acquired visual field defects (Van der Stigchel, Nijboer, Bergsma, Abegg \& Barton, 2010). Although it was already suggested by Deubel and colleagues (1988) that the global effect is modulated by visual cortical processes, there had been one other report of this effect in a patient with a visual field defect (Barbur, Forsyth \& Findlay, 1988). In the well-known blindsight patient G.Y., a global effect was found when both the target and the non-target were in the 'blind' field, although it has to be noted that it was said that G.Y. was 'aware' of these targets. In the study by Van der Stigchel and colleagues, the non-target was presented in the blind field of the patients and the target in the intact field. This set-up enabled the investigation whether visual information presented in the blind field is still processed and can influence the endpoint of an eye movement to a target presented in the intact part of the visual field. Careful mapping of the visual field defects and strict fixation control ensured that the distractor was presented in the blind part of the visual field, whereas the target was presented in the intact field. The hypothesis was tested that, in the absence of retinogeniculostriate processing, residual visual processing ('blindsight') may still be detected by measuring saccade endpoints. Whereas the global effect in response to a non-target in the intact field was present in all patients, the results for distractors in the blind field were mixed, with a shift of the endpoint away from the non-target in two out of three patients (Van der Stigchel et al., 2010). In the third patient no effect was observed. This mixed results are in line with an earlier study in which the analysis of the full trajectory of the eye movement in two hemianopic patients showed mixed results when the target and the non-target were closely aligned (Van der Stigchel, van Zoest, Theeuwes \& Barton, 2008). So, in absence of an intact retinogeniculostriate pathway, projecting from the retina to the lateral geniculate nucleus to the primary visual cortex, modulations of the saccade endpoint by a nontarget in the blind field can still be observed. It is currently unclear, however, what determines whether 
these effects of residual visual processing manifest themselves in a given patient.

\section{The global effect in reading}

Because eye movements play an important role in reading, a number of studies have investigated the influence of the mechanisms underlying the global effect during reading. During typical reading, observers make horizontal saccades to a target word in the presence of multiple foveal and peripheral stimuli. Using reading-like stimuli, it can be investigated whether these foveal and peripheral stimuli influence the endpoint of the eye movement to the target word.

The first two studies that used written material to investigate the global effect were performed by Jacobs (1987) and Coëffé \& O'Regan (1987). Although both studies used letter strings, the task was not an actual reading task, but a detection task of a target letter present within a letter string. A reading task in which two words were presented either simultaneously or in sequencewas used by Vitu (1991). Also here, the eye's initial landing position in a test word was affected by the presence of other words or stimuli in the peripheral visual field: the position where the eye landed corresponded to the location with the highest relative saliency in the visual field ('the weighted centre of gravity'). For instance, in one of the experiments, distractor stimuli were presented above or below the first word of a word pair. Results showed that the initial landing position of the eyes was positioned in the direction of these stimuli, but only when they were aligned with the central or end part of the word. These results clearly show that the global effect is also present during reading.

A follow-up study of Vitu and colleagues extended these findings by showing that stimuli that are displayed in the central foveal region fail to elicit a global effect, in contrast to stimuli outside the central foveal region (Vitu et al., 2006). This 'foveal dead zone' was observed only when the letter string presented at fixation was shorter than three letters (see also Vitu, 2008). This finding was explained by the presence of fixation neurons in the rostral pole of the superior colliculus, which discharge during fixation (Munoz \& Wurtz, 1993). When short letter strings are presented at fixation, this information is projected to only the fixation neurons which then fail to elicit the activity necessary to elicit a global effect. This will be explained further in the last part of this review.

\section{The global effect and perception}

One might argue that the global effect is simply a perceptual phenomenon, caused by the fact that observers are not able to correctly identify the appropriate target location. This does not appear to be the case. In the earlier studies of the global effect it was already established that observers are able to discriminate the target from the non-targets (Coren \& Hoenig, 1972). Furthermore, the global effect was also observed in a task in which more precise fixation was required, i.e. to determine whether there was a small gap in one of the squares (Findlay, 1982).

There is evidence, however, that the perception of the stimuli does influence the strength of the global effect. A direct comparison between the effect of a non-target on perception and the oculomotor system was performed by Eggert and colleagues (Eggert, Sailer, Ditterich \& Straube, 2002). In this experiment, participants had to indicate the point of subjective alignment of two sequentially presented peripheral targets, while a distractor was presented simultaneously. Whereas there was no effect of the distractor on the perceptual task, the saccade endpoint was positioned in the direction of the distractor. This was especially the case when saccades with a short latency were executed. The global effect is therefore not a perceptual phenomenon caused by a shift of the perceived location of the target in space. This shows that the mechanisms underlying the global effect of eye movements differ from those underlying spatial perception.

It has been suggested that the global effect is a strategy to allow rapid detection in the periphery. In a study by Jacobs (1987), participants were required to make an eye movement to a target letter presented in a string of variable lengths. Besides the target letter, the remaining letters of the two presented strings were ' $x$ 's. Results showed that the eyes landed near the middle of the string, irrespective of the target location. Only when saccade latency was greatly prolonged, saccades were accurately initiated to the target location. These results led to the suggestion that short-latency saccades are programmed to land near the middle of the string, not to land on the target itself. The global effect is in this 
explanation a by-product of an efficient strategy to quickly place the gaze onto the string, after which a second eye movement is made to identify the target.

The studies discussed until now have used simple stimuli presented on a uniform background. Uniform backgrounds might be considered artificial, as our daily visual world is generally much more complex. It is therefore important to validate that the global effect can also be observed using more complex, ecologically valid, stimuli. Indeed, the global effect was shown to be present when stimuli defined by texture differences were presented on a structured background (Deubel et al., 1988). The stimuli required foreground/background segregation, therefore constituting a more complex visual environment.

Due to the presence of the global effect in more complex visual situations, subsequent studies have used the global effect as an index to measure saliency of different visual characteristics. In these studies, it is assumed that the more salient a certain stimulus is, the stronger it will contribute to the global effect. For instance, it was found that positive and negative contrast elements elicit an equally large global effect and that the boundary of an object is an important contributor to determine the amount of salience of an object (Findlay et al., 1993). Furthermore, the global effect was found to be stronger for targets defined by shape than targets defined by color (Findlay \& Gilchrist, 1997). This finding was explained by the fact that it is more difficult to discriminate shape than color. The global effect is therefore observed throughout the latency distribution for shape stimuli, whereas the target decision for color targets is made earlier in the latency distribution, resulting in an overall weaker global effect.

The previous studies have used stimuli which consisted of small elements presented in space. This might not be ecologically valid, however, because our complex visual environment usually consists of spatially extended objects. Studies that have used spatially extended objects have used various types of stimuli and consistently observed that the eyes land on the centre of gravity in these items (He \& Kowler, 1991, Kowler \& Blaser, 1995). Examples of these stimuli are random dot targets (McGowan, Kowler, Sharma \& Chubb, 1998), large letters (Vishwanath \& Kowler, 2003) and line drawings which participants needed to inspect in order to count the number of corners (Guez, Marchal, Le
Gargasson, Grall \& O'Regan, 1994). This latter study showed that the eyes landed at a position near the centre of gravity of the corner configurations. Furthermore, when observers were presented with faces, they initially fixated the geometric centre of the face, independent of face view (Bindemann, Scheepers \& Burton, 2009).

One interesting application of the centre of gravity account relates to the perception of visual illusions (de Grave, Smeets \& Brenner, 2006, Gilster \& KuhtzBuschbeck, 2010). By measuring eye movements in response to a visual illusion, it can be investigated whether the action system and the perceptual system use different or common codes. Because the saccade endpoint reflects the centre of gravity for the action system, this endpoint can be compared to the observed centre for the perceptual system. Although one study did not observe a similar perceptual and motor effect for some eye movement directions (de Grave et al., 2006), a recent study showed that perception and action appear to share a common internal representation (Gilster \& KuhtzBuschbeck, 2010).

Finally, the centre of gravity account has been used in realistic search task in which real objects needed to be found in a pseudorealistic scene (Zelinsky, Rao, Hayhoe \& Ballard, 1997). Also here, participants generally first made an eye movement to the global centre of the configuration instead of to individual items.

\section{The global effect in visual search}

Because eye movements reflect the current point of interest of an observer, eye movements have been frequently measured during visual search tasks. Eye movements allow insight in the time course and the strategies during search behavior. Various studies have observed that the saccade endpoint towards the search target is deviated in the direction of non-targets. For instance, Findlay (1997) investigated whether an accurate saccade could be executed to a target in the presence of multiple non-targets. On some trials, double targets were presented. In this situation, the first saccade sometimes landed at an intermediate position between the two targets (see also Viviani \& Swensson, 1982). Also in the oculomotor capture task, in which a task-irrelevant onset is presented, a global effect was observed for the fastest saccades when the target and the onset were closely aligned (Godijn \& Theeuwes, 2002). A similar effect has 
also been observed in monkeys (McPeek \& Keller, 2001) and during a visual search task in which multiple eye movements were executed (Findlay \& Brown, 2006).

A recent study investigated the global effect using a modified version of the oculomotor capture task (Van der Stigchel et al., 2011). As it is known that participants frequently perform an erroneous saccade towards the onset distractor in the oculomotor capture task ('a capture saccade') the authors investigated whether the global effect also occurs for eye movements executed to distracting elements. To this end, the task was made such that on a subset of trials, two distractors were presented simultaneously. When the two distractors were closely aligned, erroneous eye movements were initiated to a location in between the two distractors. Even though to a lesser extent, this effect was also present when the two distractors were presented further apart. In a second experiment, the global effect was investigated for eye movements in the presence of two targets. A strong global effect was observed when two targets were presented closely aligned, while this effect was absent when the targets were further apart. The results of this study therefore showed that there is also a global effect when saccades are captured by distractors. This 'capture global' effect is different from the traditional global effect that occurs when two targets are presented because the global effect of capture saccades was also quite strong for remote elements.

\section{The underlying mechanisms of the global effect}

After reviewing the studies on the global effect, we will propose a framework that can account for the discussed findings. As mentioned earlier, the global effect has traditionally been explained in terms of a centre of gravity-account which states that the saccade endpoint reflects the relative saliency of the different elements in the visual scene (Coren \& Hoenig, 1972). When considering the results of the discussed studies, it has to be concluded that this account can not fully explain the observed phenomena. For instance, the global effect is not simply determined by the (low-level) visual information in the visual scene, but is also influenced by higher-order signals, such as target expectancy (e.g. Coeffe \& O'Regan, 1987). This indicates that there is more to the global effect than merely a computation on the basis of visual information. Furthermore, even information that is no longer visually presented, like an element that has to be kept in memory (e.g. Herwig et al., 2010), is known to influence the saccade landing position.

For these reasons, the centre of gravity-account needs to be extended. The global effect appears to be better explained in terms of a weighted average of activity in a saccade map. This explanation is in line with various models of saccade generation which have assumed that target selection is the result of competitive interactions among groups of neurons coding for possible target locations on a common map (Godijn \& Theeuwes, 2002, Kopecz, 1995, McSorley, Haggard \& Walker, 2004, Meeter, Van der Stigchel \& Theeuwes, 2010, Trappenberg, Dorris, Munoz \& Klein, 2001). In this (retinotopic) saccade map, possible saccade goals are represented by peaks of activity. A peak of activity can be evoked by the presentation of a visual stimulus, but can also be modulated by a task instruction to make an eye movement to this element. In line with the vector theory of Tipper and colleagues (Tipper, Howard \& Jackson, 1997), the saccade endpoint is then determined by the weighted average of the activity present in this saccade map on the moment the eye movement is initiated. In case of the global effect, there are two peaks of activity and the weighted average is positioned in between the two peaks. There appears to be only a limited area in which this averaging occurs. Clearly, the weighted average is based on a restricted region in the motor map.

The activity in the saccade map is determined by the interaction between bottom-up (or stimulus-driven) and top-down (or task-driven) information (Ludwig \& Gilchrist, 2002, Ludwig \& Gilchrist, 2003, van Zoest, Donk \& Theeuwes, 2004). Bottom-up information reflects the influence from the outside world, every image that falls on our retina. Top-down information reflects all intentions and goals that one might have at a certain moment. As visual attention and eye movement are strongly related (Rizzolatti, Riggio \& Sheliga, 1994, Van der Stigchel \& Theeuwes, 2007), both types of information reflect the same constructs as used in the attention literature (for a review, see Van der Stigchel, Belopolsky, Peters, Wijnen, Meeter \& Theeuwes, 2009). The continuous competition between these two types of information has to be resolved in order to execute an eye movement. Behavioral studies have shown that bottom- 
up information is dominant early in the selection process, whereas top-down information can influence the selection process with increasing latency (Ludwig \& Gilchrist, 2002, Ludwig \& Gilchrist, 2003, van Zoest et al., 2004). Therefore, saccades with a short latency are mostly modulated by bottom-up information, which explains why the global effect is stronger for saccades with a short latency. In this situation, a saccade is executed to an intermediate location because no target has been selected yet. When top-down information has selected one of the two elements as the target, the peak associated with the target will be stronger than the peak associated with the non-target, resulting in a weaker global effect.

The motor map in which this competition is resolved is often thought to be located in the intermediate layers of the superior colliculus (SC) (Schall, 1991, Sparks \& Hartwich-Young, 1989). This mid-brain structure contains a retinotopically-organized map in which neural activity is correlated with target selection (McPeek \& Keller, 2004, Wurtz, Goldberg \& Robinson, 1980). The $\mathrm{SC}$ integrates input from many cortical areas such as the Frontal Eye Fields (FEF), the Supplementary Eye Fields (SEF), the posterior parietal cortex and occipital visual areas (Munoz, 2002) and sends the outcome of this integration process to the brainstem premotor circuitry where the eye movement is programmed (Moschovakis, 1996). Cortical processes can therefore influence the global effect through the projections to the SC (Deubel et al., 1988). In line with the weighted average account explained above, neurophysiological recordings in the SC have shown that the largest neural activity was positioned at the sites of the two visual stimuli in trials in which the global effect was observed (Edelman \& Keller, 1998).

Various models which have implemented a motor map have been able to account for the global effect. For instance, the models by Van Opstal and Van Gisbergen (1989) and Findlay and Walker (1999) showed that saccade averaging can be explained by implementing population coding of saccade target positions in a motor map like the SC. A recent computational model of the SC based on these principles extended these findings by accounting for the effect of saccade latency on the global effect (Meeter et al., 2010). At both target locations, there was a peak of activity that directed the saccade towards the endpoint coded by the peak locations, resulting in a saccade directed in between the two locations. When two stimuli were presented closely aligned, saccades with a short latency tended to go to the centre in between the target and distractor. With increasing saccade latency, saccades were directed more and more towards the target.

This explanation can account for the various observations discussed in this review by the assumption that the saccade endpoint will be positioned in the direction of the stronger of the two peaks. For instance, when one element is larger or has a stronger intensity than the other, this element will evoke a stronger peak, resulting in an eye movement which is directed towards the larger element. Due to the cortical magnification factor, the element closer to fixation will result in a stronger peak than the other element, leading to a shift of the saccade endpoint towards the element closer to fixation. With respect to auditory information, it is known that the SC also codes for information from other modalities (Meredith \& Stein, 1986), which explains the global effect when a visual tone is presented together with a visual stimulus.

The explanation of the global effect in terms of the averaging of activity in a saccade map can also be applied to other behavioral phenomena. For instance, in the 'double step paradigm' (Becker \& Jürgens, 1979), a single target is shortly presented but is displaced while a saccade is programmed. This situation results in a saccade which is initiated towards a location in between the original location of the target and the final location of the target. Previous researchers have already suggested that these saccades may result from the spatial averaging of activity in a dynamically changing saccade map (e.g., Deubel et al., 1984).

Especially with the paradigm in which two elements are presented, it is relatively straightforward to explain the various findings associated with the global effect. As discussed, the term 'global effect' has also been used to account for findings in paradigm using more natural displays. A recent computation model by Zelinsky (2008) aimed to account for the oculomotor behavior during the search for a target in a more complex visual scene. In this model, the location of the next fixation is determined by the activity pattern in a target map. The location of the saccade endpoint is computed by the weighted spatial average of the activity in this target map. The behavior of the model resembled the data pattern observed in the paradigms discussed above, showing averaging in the direction of the strongest activity in the target map. 


\section{Conclusion}

Because the exact landing position of an eye movement has shown to reflect the interaction between visual and higher-order information, the global effect is an interesting measure to gain more insight in the mechanisms underlying oculomotor behavior. In future research, application of this measure in special populations, like brain lesion patients and psychiatric disorders, might constitute a valuable tool to provide valuable information about the functioning of the oculomotor system in these groups. Future studies on the effect of aging on the global effect might tell us more about the development of the oculomotor system.

\section{Acknowledgements}

This research was funded by two grants from NWO (Netherlands organization for Scientific Research): grant 451-09-019 to SvdS and grant 451-10-013 to TCWN.

We thank Françoise Vitu and an anonymous reviewer for their helpful comments on an earlier version of this review.

\section{References}

Aitsebaomo, A.P., \& Bedell, H.E. (2000). Saccadic and psychophysical discrimination of double targets. Optometry and Vision Science, 77 (6), 321-330.

Arai, K., McPeek, R.M., \& Keller, E.L. (2004). Properties of Saccadic Responses in Monkey When Multiple Competing Visual Stimuli Are Present. $J$ Neurophysiol, 91 (2), 890-900.

Barbur, J.L., Forsyth, P.M., \& Findlay, J.J. (1988). Human saccadic eye movements in the absence of the geniculocalcarine projection. Brain, 111, 63-82.

Becker, W., \& Jürgens, R. (1979). An analysis of the saccadic system by means of double step stimuli. Vision Research, 19, 967-983.

Bindemann, M., Scheepers, C., \& Burton, A.M. (2009). Viewpoint and center of gravity affect eye movements to human faces. Journal of Vision, 9 (2), 1-16.

Chou, I., Sommer, M.A., \& Schiller, P.H. (1999). Express averaging saccades in monkeys. Vision Research, 39, 4200-4216.
Coeffe, C., \& O'Regan, J.K. (1987). Reducing the influence of non-target stimuli on saccade accuracy: Predictability and latency effects. Vision Research, 27, 227-240.

Cohen, M.E., \& Ross, L.E. (1978). Latency and accuracy characteristics of saccades and corrective saccades in children and adults. Journal of Experimental Child Psychology, 26, 517-527.

Coren, S., \& Hoenig, P. (1972). Effect of non-target stimuli on the length of voluntary saccades. Perceptual and Motor Skills, 34, 499-508.

de Grave, D.D.J., Smeets, J.B.J., \& Brenner, E. (2006). Why are saccades influenced by the Brentano illusion? Experimental Brain Research, 175, 177-182.

Deubel, H., Findlay, J.M., Jacobs, A.M., \& Brogan, D. (1988). Saccadic eye movements to targets defined by structure differences. In: G. Luer, U. Lass, \& J. Shallo-Hoffman (Eds.), Eye movement research: Physiological and psychological aspects (pp. 107145): C.J. Hogrefe.

Deubel, H., Wolf, W., \& Hauske, M. (1984). The evaluation of the oculomotor error signal. In: A.G. Gale, \& F.W. Johnson (Eds.), Theoretical and applied aspects of oculomotor research (Elsevier.

Dorris, M.C., Pare, M., \& Munoz, D.P. (1997). Neuronal activity in monkey superior colliculus related to the initiation of saccadic eye movements. Journal of Neuroscience, 17, 8566-8579.

Edelman, J.A., \& Keller, E.L. (1998). Dependence on target configuration of express saccade-related activity in the primate superior colliculus. Journal of Neurophysiology, 80, 1407-1426.

Eggert, T., Sailer, U., Ditterich, J., \& Straube, A. (2002). Differential effect of a distractor on primary saccades and perceptual localization. Vision Research, 42, 2969-2984.

Findlay, J.M. (1982). Global visual processing for saccadic eye movements. Vision Research, 22, 10331045.

Findlay, J.M. (1997). Saccade target selection during visual search. Vision Research, 37, 617-631.

Findlay, J.M., \& Blythe, H.I. (2009). Saccade target selection: Do distractors affect saccade accuracy? Vision Research, 49, 1267-1274.

Findlay, J.M., Brogan, D., \& Wenban-Smith, M.G. (1993). The spatial signal for saccadic eye movements emphasizes visual boundaries. Perception \& Psychophysics, 53 (6), 633-641.

Findlay, J.M., \& Brown, V. (2006). Eye scanning of multi-element displays: II Saccadic planting. Vision Research, 46, 216-227. 
Findlay, J.M., \& Gilchrist, I.D. (1997). Spatial scale and saccade programming. Perception, 26, 1159-1167.

Findlay, J.M., \& Kapoula, Z. (1992). Scrutinization, spatial attention, and the spatial programming of saccadic eye movements. The Quarterly Journal of Experimental Psychology, 45A (4), 633-647.

Findlay, J.M., \& Walker, R. (1999). A model of saccade generation based on parallel processing and competitive inhibition. Behavioral and Brain Sciences, 22, 661-721.

Fischer, B., \& Weber, H. (1993). Express saccades and visual attention. Behavioral and Brain Sciences, 16, 553-610

Frens, M.A., Van Opstal, A.J., \& Van der Willigen, R.E. (1995). Spatial and temporal factors determine auditory-visual interactions in human saccadic eye movements. Perception \& Psychophysics, 57, 802816.

Gielen, C., van den Heuvel, P.J., \& van Gisbergen, J.A.M. (1984). Coordination of fast eye and hand movements in a tracking experiment. Experimental Brain Research, 56, 154-161.

Gilster, R., \& Kuhtz-Buschbeck, J.P. (2010). The MüllerLyer illusion: investigation of a center of gravity effect on the amplitudes of saccades. Journal of Vision, 10 (1), 1-13.

Godijn, R., \& Theeuwes, J. (2002). Programming of endogenous and exogenous saccades: evidence for a competitive integration model. Journal of Experimental Psychology: Human Perception and Performance, 28 (5), 1039-1054.

Guez, J.-E., Marchal, P., Le Gargasson, J.-F., Grall, Y., \& O'Regan, J.K. (1994). Eye fixations near corners: Evidence for a centre of gravity calculation based on contrast, rather than luminance or curvature. Vision Research, 34 (12), 1625-1635.

He, P., \& Kowler, E. (1989). The role of location probability in the programming of saccades: Implications for "center-of-gravity" tendencies. Vision Research, 29, 1165-1181.

He, P., \& Kowler, E. (1991). Saccadic localization of eccentric forms. Journal of the Optical Society of America, 8, 440-449.

Herwig, A., Beisert, M., \& Schneider, W.X. (2010). On the spatial interaction of visual working memory and attention: Evidence for a global effect from memoryguided saccades. Journal of Vision, 10 (5), 1-10.

Jacobs, A.M. (1987). On localization and saccade programming. Vision Research, 27 (11), 1953-1966.
Kaufman, L., \& Richards, W. (1969). Spontaneous fixation tendencies for visual forms. Perception \& Psychophysics, 2, 359-362.

Kopecz, K. (1995). Saccadic reaction times in gap/overlap paradigms: A model based on integration of intentional and visual information on neural dynamic fields. Vision Research, 35, 2911-2925.

Kowler, E., \& Blaser, E. (1995). The accuracy and precision of saccades to small and large targets. Vision Research, 35, 1741-1754.

Levy-Schoen, A. (1969). Determination et latence de la reponse oculo-motrice a deux stimulus simultanes ou successifs selon leur excentricite relative. Annee psychologique, 69, 373-392.

Ludwig, C.J.H., \& Gilchrist, I.D. (2002). Stimulus-driven and goal-driven control over visual selection. Journal of Experimental Psychology: Human Perception and Performance, 28 (4), 902-912.

Ludwig, C.J.H., \& Gilchrist, I.D. (2003). Target similarity affects saccade curvature away from irrelevant onsets. Experimental Brain Research, 152, 60-69.

McGowan, J.W., Kowler, E., Sharma, A., \& Chubb, C. (1998). Saccadic localization of random dot targets. Vision Research, 38 (6), 895-909.

McPeek, R.M., \& Keller, E.L. (2001). Short-term priming, concurrent processing, and saccade curvature during a target selection task in the monkey. Vision Research, 41 (6), 785-800.

McPeek, R.M., \& Keller, E.L. (2004). Deficits in saccade target selection after inactivation of superior colliculus. Nature Neuroscience, 7, 757-763.

McSorley, E., \& Findlay, J.M. (2003). Saccade target selection in visual search: Accuracy improves when more distractors are present. Journal of Vision, 3, 877-892.

McSorley, E., Haggard, P., \& Walker, R. (2004). Distractor modulation of saccade trajectories: spatial separation and symmetry effects. Experimental Brain Research, 155, 320-333.

Meeter, M., Van der Stigchel, S., \& Theeuwes, J. (2010). A competitive integration model of exogenous and endogenous eye movements. Biological Cybernetics, 102, 271-291.

Menz, C., \& Groner, R. (1987). Saccadic programming with multiple targets under different task conditions. In: J.K. O'Regan, \& A. Levy-Schoen (Eds.), Eye movements: From physiology to cognition (pp. 95103). North-Holland: Elsevier Science Publishers. 
Meredith, M.A., \& Stein, B.E. (1986). Visual, auditory, and somatosensory convergence on cells in superior colliculus results in multisensory integration. Journal of Neurophysiology, 56 (3), 640-662.

Moschovakis, A.K. (1996). The superior colliculus and eye movement control. Current Opinions in Neurobiology, 6, 811-816.

Munoz, D.P. (2002). Commentary: saccadic eye movements: overview of neural circuitry. Progress in Brain Research, 140, 89-96.

Munoz, D.P., \& Wurtz, R.H. (1993). Fixation cells in monkey superior colliculus. II. Reversible activation and deactivation. Journal of Neurophysiology, 70 (2), 576-589.

Ottes, F.B., Van Gisbergen, J.A.M., \& Eggermont, J.J. (1985). Latency dependence of colour-based target vs nontarget discrimination by the saccadic system. Vision Research, 25, 849-862.

Ottes, F.P., van Gisbergen, J.A.M., \& Eggermont, J.J. (1984). Metrics of saccade responses to visual double stimuli: two different modes. Vision Research, 24 (10), 1169-1179.

Pare, M., \& Munoz, D.P. (1996). Saccadic reaction time in the monkey: advanced preparation of oculomotor programs is primarily responsible for express saccade occurrence. J Neurophysiol, 76 (6), 3666-3681.

Pitts, W., \& McCulloch, W.S. (1947). How we know universals. The perception of auditory and visual forms. Bulletin of Mathematical Biophysics, 9, 127147.

Rizzolatti, G., Riggio, L., \& Sheliga, B.M. (1994). Space and selective attention. In: C. Umilta, \& $\mathrm{M}$. Moscovitch (Eds.), Attention and Performance XIV (pp. 231-265): MIT Press.

Sailer, U., Eggert, T., Ditterich, J., \& Straube, A. (2002). Global effect of a nearby distractor on targeting eye and hand movements. Journal of Experimental Psychology: Human Perception and Performance, 28 (6), 1432-1446.

Schall, J.D. (1991). Neuronal basis of saccadic eye movements. In: A.G. Leventhal (Ed.) Vision and Visual Dysfunction. Volume 4: The Neural Basis of Visual Function (pp. 388-442). London: Macmillan Press.

Sparks, D.L., \& Hartwich-Young, R. (1989). The deeper layers of the superior colliculus. In: R.H. Wurtz, \& M.E. Goldberg (Eds.), The Neurobiology of Saccadic Eye Movements (pp. 213-255): Elsevier Science Publishers.
Tipper, S.P., Howard, L.A., \& Jackson, S.R. (1997). Selective reaching to grasp: evidence for distractor interference effects. Visual Cognition, 4, 1-38.

Trappenberg, T.P., Dorris, M.C., Munoz, D.P., \& Klein, R.M. (2001). A model of saccade initiation based on the competitive integration of exogenous and endogenous signals in the superior colliculus. Journal of Cognitive Neuroscience, 13 (2), 256-271.

Van der Stigchel, S., Belopolsky, A.V., Peters, J.C., Wijnen, J.G., Meeter, M., \& Theeuwes, J. (2009). The limits of top-down control of visual attention. Acta Psychologica, 132 (2), 201-212.

Van der Stigchel, S., de Vries, J., Bethlehem, R., \& Theeuwes, J. (2011). A global effect of capture saccades. Experimental Brain Research, 210 (57-65)

Van der Stigchel, S., Meeter, M., \& Theeuwes, J. (2007). The spatial coding of the inhibition evoked by distractors. Vision Research, 47 (2), 210-218.

Van der Stigchel, S., Mulckhuyse, M., \& Theeuwes, J. (2009). Eye cannot see it: The interference of subliminal distractors on saccade metrics. Vision Research, 49, 2104-2109.

Van der Stigchel, S., Nijboer, T.C.W., Bergsma, D.P., Abegg, M., \& Barton, J.J.S. (2010). Anomalous global effects induced by 'blind' distractors in visual hemifield defects Brain \& Cognition, 74, 66-73.

Van der Stigchel, S., \& Theeuwes, J. (2005). Relation between saccade trajectories and spatial distractor locations. Cognitive Brain Research, 25 (2), 579-582.

Van der Stigchel, S., \& Theeuwes, J. (2007). The relationship between covert and overt attention in endogenous cueing. Perception \& Psychophysics, 69 (5), 719-731.

Van der Stigchel, S., van Zoest, W., Theeuwes, J., \& Barton, J.J.S. (2008). The influence of 'blind' distractors on eye movement trajectories in visual hemifield defects. Journal of Cognitive Neuroscience, 20 (11), 2025-2036.

Van Opstal, A.J., \& Van Gisbergen, J.A.M. (1989). A nonlinear model for collicular spatial interactions underlying the metrical properties of electrically elicited saccades. Biological Cybernetics, 60, 171183.

van Zoest, W., Donk, M., \& Theeuwes, J. (2004). The role of stimulus-driven and goal-driven control in saccadic visual selection. Journal of Experimental Psychology: Human Perception and Performance, 30 (4), 746-759.

Vishwanath, D., \& Kowler, E. (2003). Localization of shapes: eye movements and perception compared. Vision Research, 43, 1637-1653. 
Vitu, F. (1991). The existence of a center of gravity effect during reading. Vision Research, 31, 1289-1313.

Vitu, F. (2008). About the global effect and the critical role of retinal eccentricity: Implications for eye movements in reading. Journal of Eye Movement Research, 2 (3), 1-18.

Vitu, F., Lancelin, D., Jean, A., \& Farioli, F. (2006). Influence of foveal distractors on saccadic eye movements: A dead zone for the global effect. Vision Research, 46, 4684-4708.

Viviani, P., \& Swensson, R.G. (1982). Saccadic eye movements to peripherally discriminated visual targets. Journal of Experimental Psychology: Human Perception and Performance, 8 (1), 113- 126.

Walker, R., Deubel, H., Schneider, W.X., \& Findlay, J.M. (1997). Effect of remote distractors on saccade programming: evidence for an extended fixation zone. Journal of Neurophysiology, 78 (2), 1108-1119.

Wurtz, R.H., Goldberg, M.E., \& Robinson, D.L. (1980). Behavioral modulation of visual responses in the monkey: Stimulus selection for attention and movement. Progress in Psychobiology and Physiological Psychology, 9, 43-83.

Zelinsky, G.J. (2008). A theory of eye movements during target acquisition. Psychological Review, 115 (4), 787-835.

Zelinsky, G.J., Rao, R.P.N., Hayhoe, M.M., \& Ballard, D.H. (1997). Eye movements reveal the spatiotemporal dynamics of visual search. Psychological Science, 8 (6), 448-453. 